13

\title{
Двухволновая лазерная стереолитография для создания ИК сенсоров для поверхностно-усиленной спектроскопии*
}

\author{
() А.Г. Витухновский ${ }^{1,2}$, Р.Д. Звагельский ${ }^{1}$, Д.А. Колымагин ${ }^{1}$, А.В. Писаренко ${ }^{1}$, Д.А. Чубич1 \\ ${ }^{1}$ Московский фризико-технический институт (государственный университет), \\ 141700 Долгопрудный, Россия \\ ${ }^{2}$ Физический институт им. П.Н. Лебедева РАН, \\ 119991 Москва, Россия \\ e-mail: chubich.conference@gmail.com
}

Поступила в редакцию 24.09.2018 г.

\begin{abstract}
Представлены результаты применения метода двухфотонной фемтосекундной лазерной фотополимеризации для создания структур для чувствительных сенсоров ИК диапазона. Проведено сопоставление двух методов создания сенсоров: двухволновой лазерной стереолитографии и электронно-лучевой литографии. Продемонстрирована возможность применения полученных структур для исследования эффекта поверхностноусиленного ИК поглощения (SEIRA) со STED-совместимым олигомером Pentaerythinol Tetraacrylate (PETTA) в качестве аналитического слоя.
\end{abstract}

DOI: $10.21883 /$ OS.2019.01.47055.271-18

\section{Введение}

В последние десятилетия большое внимание привлекает проблема усиления взаимодействия электромагнитного излучения с веществом. Важные результаты в этом направлении были достигнуты в рамках спектроскопических исследований. Так, открытая в 1977 г. спектроскопия гигантского комбинационного рассеяния [1] или Surface Enhancement of Raman Scattering (SERS в английской транскрипции) позволила наблюдать существенное усиление сигнала комбинационного рассеяния. Это явление обусловлено адсорбцией молекул на поверхности металлов, связанной с сильными межмолекулярными взаимодействиями, обусловленными эффектом Рамана [2] и плазмонными эффектами [3]. Недавние разработки в области спектроскопии гигантского комбинационного рассеяния позволили значительно повысить чувствительность измерений и обнаружить новые явления [4,5]. Также поверхностно усиленное ИК поглощение (SEIRA), открытое в 1980 г. [6], позволило существенно увеличить сечение поглощения молекул в ИК диапазоне при исследованиях методом фурьеспектроскопии. Применение этого метода важно для химии, биохимии и биофизики [7].

Для контролируемой реализации отмеченных спектральных методов требуется создание плазмонных наноструктур как планарной геометрии, так и трехмерной. В настоящее время помимо метода создания наноструктур традиционной электронно(ионной)-лучевой литографией [8] бурно развивается метод прямого лазерного письма (Direct Laser Writing-DLW в английской транскрипции) [9-11]. Методы DLW-литографии могут быть улучшены аналогично с методами люминесцентной

* XIII International Conference on Hole Burning, Single Molecule, and Related Spectroscopies: Science and Applications (HBSM-2018), August 6-12, 2018, Suzdal-Moscow, Russia. микроскопии. Например, благодаря идее использования гасящего излучения (STED - Stimulated Emission Depletion) Штефана Хэлла (S. Hell, нобелевская премия по химии в 2014 г. [12]) в оптической микроскопии было продемонстрировано разрешение $2.4 \mathrm{~nm}$ [13], что меньше длины волны возбуждения на два порядка. Данную идею возможно использовать и для лазерной литографии. Таким образом, сейчас авторами используется оригинальная объединенная методика STED-DLW для создания наноразмерных SEIRA-активных структур (сенсоров).

В работе представлено описание используемой методики STED-DLW для создания сенсорных плазмонных структур и сопоставление с результатами использования электронно-лучевой литографии для создания аналогичных структур. Продемонстрирована возможность применения полученных плазмонных структур для исследования эффекта SEIRA со STED-совместимым олигомером PETTA в качестве аналитического слоя.

\section{Экспериментальная часть}

Метод DLW основан на двухфотонной полимеризации молекул фоторезиста [11], который представляет собой смесь фотоинициатора и олигомера. Первый компонент является фотоактивным веществом с большим сечением поглощения, возбуждение которого инициируется с помощью эффекта двухфотонного поглощения (рис. 1,a) излучением фемтосекундного лазера, обеспечивающего высокую плотность мощности излучения вблизи точки фокусировки излучения. После оптического возбуждения происходит релаксация молекулы фотоинициатора на нижний колебательный подуровень $S_{1}$ и интеркомбинационный переход на нижний триплетный уровень $T_{1} \mathrm{c}$ последующим образованием свободных радикалов $R^{*}$. 
$a$

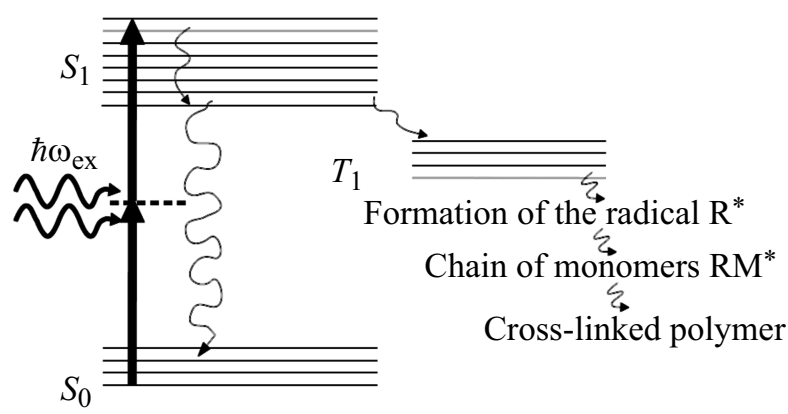

$b$

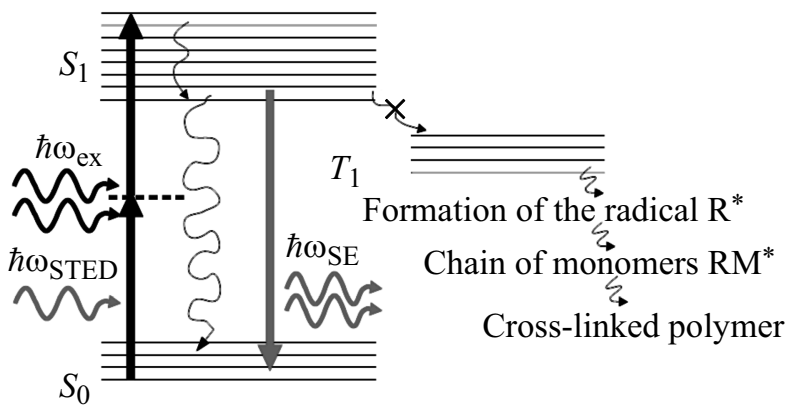

Рис. 1. Энергетическая диаграмма для фотополимеризации олигомера в результате $(a)$ DLW-литографии и $(b)$ STED-стереолитографии.

Второй компонент фоторезиста - это олигомер, молекулы которого последовательно присоединяются к „растущему“ радикалу $R M^{*}$. Образованная таким способом цепь является сшитым (cross-linked) полимером. В качестве олигомера использовалось соединение акрилового ряда PETTA (Pentaerythritol Tetraacrylate). В процессе двухфотонной фотополимеризации олигомер преобразуется в твердый, нерастворимый полимер, обладающий устойчивостью к механическому воздействию и являющийся диэлектриком.

Перемещение области фокусировки фемтосекундного излучения относительно слоя фоторезиста при экспозиции производится с помощью прецизионного пьезопозиционера, что дает возможность получения 3D-структур с разрешением, ограниченным размером пространственного пикселя в $200 \mathrm{~nm}$ для латерального диаметра и 800 $\mathrm{nm}$ для аксиального диаметра.

Увеличение пространственного разрешения (STED) достигается с использованием второго лазера гашения с длиной волны, попадающей в полосу люминесценции фотоинициатора и одновременно не попадающей в полосу его поглощения. Вынужденное излучение при определенных параметрах является конкурирующим с безызлучательными переходами и обедняет уровень $S_{1}$, не допуская переход на триплетный уровень $T_{1}$ (рис. $1, b$ ).

Таким образом, можно локализовать фотополимеризацию в области с диаметром гораздо меньше, чем дифракционный предел. Локализация для латерального размера осуществляется с использованием профиля гасящего излучения в виде „тора“ (donut), который реализуется вихревой фазовой пластинкой с набегом фаз в $2 \pi$. Для фоторезиста PETTA/DETC было измерено время жизни синглетного уровня $S_{1}$, которое оказалось равным $2.4 \mathrm{~ns}$, что позволило использовать непрерывный лазер гашения на длине волны $532 \mathrm{~nm}$ и использовать скорости сканирования до $10 \mathrm{~mm} / \mathrm{s}$ для STED-DLW-литографии.

Дальнейшее создание металлических структур достигается посредством металлизации полученных полимерных шаблонов. Для метализации рассматривались следующие методы: взрывная (lift-off) литография позитивными и негативными фоторезистами [14], травление ионным пучком [15], электрохимическое осаждение [16]. В данной работе наиболее эффективным методом является взрывная lift-off-металлизация.

Двухволновая стереолитография реализуется с использованием созданной оригинальной установки [17,18]. Фотополимеризация во время процесса литографии инициируется фемтосекундным излучением титан-сапфирового (Ti:Sapphire) лазера с перестраиваемой длиной волны $\lambda=740-840 \mathrm{~nm}$, длительностью импульса $50 \mathrm{fs}$ и частотой $80 \mathrm{MHz}$. Гашение полимеризации реализуется при помощи непрерывного лазерного излучения с длиной волны $532 \mathrm{~nm}$. Лазерное излучение фокусируется апохроматическим объективом с $N A=1.44$ на границу фоторезист-подложка. Пороговое значение мощности для полимеризации эталонного фоторезиста на входе в объектив составило около $6 \mathrm{~mW}$. В установке используются два типа позиционеров: шаговый для грубой подводки подложки к лазерному лучу и рисования двумерных фигур с простейшей геометрией (точность позиционирования около $10 \mathrm{~nm}$ ) и пьезопозиционер с линейной обратной связью для лазерного письма трехмерных структур со сложной геометрией (точность позиционирования около $0.5 \mathrm{~nm}$ ). Скорость пьезосканирования для полученной установки может быть установлена в диапазоне от 1 до $100 \mu \mathrm{m} / \mathrm{s}$. Был подобран состав фоторезистов и продемонстрирована запись для фоточувствительной композиции на основе олигомера pentaerythritol tetraacrylate (PETTA) и фотоинициатора 7-diethylamino3-thenoylcoumarin (DETC) на различных подложках. Принципиальная схема установки приведена на рис. 2 .

Серия полимерных шаблонов, созданных с помощью описанной выше установки, обладает достаточной периодичностью и качеством полученных элементов при создании массивов наноантенн (рис. 3,a). Серии полимерных шаблонов были реализованы на разных типах подложек: стеклянных, проводящих гибких, прозрачных в ИК диапазоне спектра электромагнитных волн. В результате морфологических исследований созданных структур было показано, что антенны имеют больший латеральный размер по сравнению с моделируемым. Это 


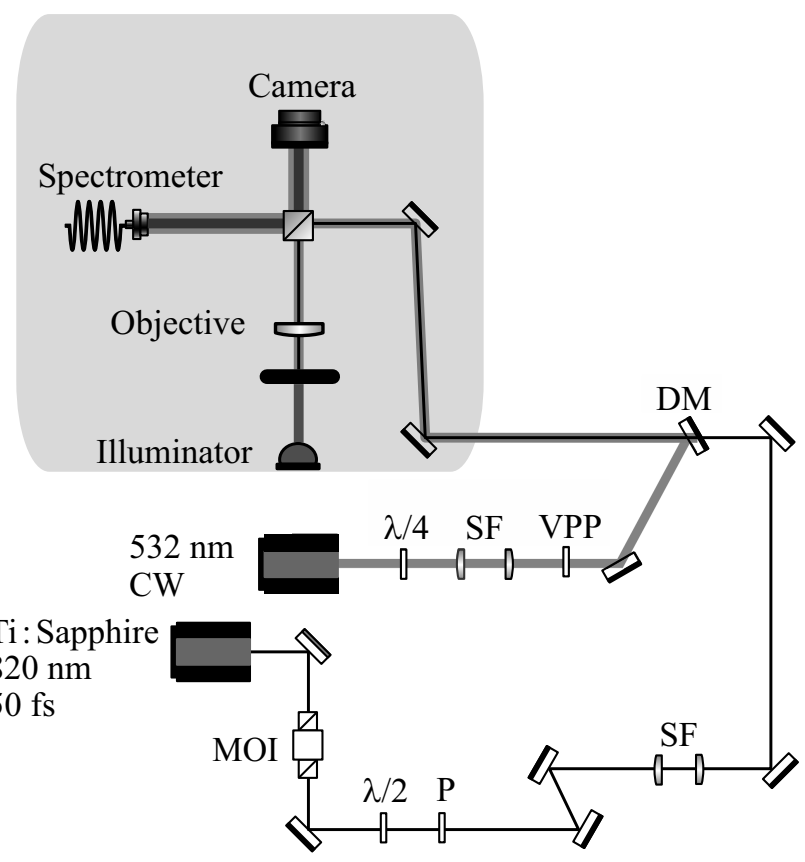

Рис. 2. Схема установки для DLW и STED-DLW стереолитографии. Возбуждение фоточувствительного олигомера реализуется посредством фемтосекундных импульсов титансапфирового лазера (Ti:Sapphire) на длине волны $820 \mathrm{~nm}$. Гашение - непрерывным излучением (CW - continuous wave) твердотельного лазера с диодной накачкой с длиной волны $532 \mathrm{~nm}$. На схеме введены следующие сокращения: $\mathrm{MOI}$ - магнитооптический изолятор; $\mathrm{P}$ - поляризатор, $\mathrm{SF}$ пространственный фильтр; VPP — вихревая фазовая пластина; $\mathrm{DM}$ - дихроическое зеркало.

связано с большим временем экспозиции в результате использования электромеханического затвора для прерывания излучения. Улучшение размерных параметров структур возможно получить при использовании акустооптического затвора.

Часть структур была подготовлена методом электронно-лучевой (e-beam) литографии с последующей взрывной lift-off-металлизацией. Размерные параметры структур, получаемых методами двухволновой лазерной стереолитографии и электронно-лучевой литографии, были выбраны одинаковыми. А именно размерные параметры структур для e-beam-литографии были определены по результатам измерений латеральных размеров линий, полученных с помощью метода двухволновой лазерной стереолитографии.

Для получения плазмонного резонанса массива плазмонных наноантенн в районе линий поглощения используемого аналитического материала подобраны геометрические и размерные параметры структур. Данные серии структур имеют вид массивов параллелепипедов общим размером $210 \times 170 \mu \mathrm{m}$ и массивов $C$ образных структур размером $110 \times 110 \mu \mathrm{m}$, являющихся разомкнутыми кольцевыми резонаторами. Для массива параллелепипедов (рис. 3, $b$ ) использовались следующие параметры: длина штриха $l=2$ и $2.3 \mu \mathrm{m}$, ширина штриха $w=150 \mathrm{~nm}$, расстояние между штрихами $\Delta x=2 \mu \mathrm{m}$ (вдоль), $\Delta y=1 \mu \mathrm{m}$ (поперек). Использовались подложки $\mathrm{CaF}_{2}$, прозрачные в ИК диапазоне. Далее по шаблону производилась электронно-лучевая литография с резистом AR-P и тонким адгезивным слоем хрома. Затем напылялся $80 \mathrm{~nm}$ слой золота и осуществлялась операция lift-off в изопропиловом спирте и $\mathrm{N}$-метил-2-пирролидоне. Морфология полученных структур была исследована на атомно-силовом микроскопе Ntegra Spectra в контактном и полуконтактном режимах, а также на сканирующем электронном микроскопе и темнопольном оптическом микроскопе.

Создание тонкого аналитического слоя (олигомер PETTA) для исследования эффекта SEIRA на плазмонных структурах осуществлялось с помощью метода

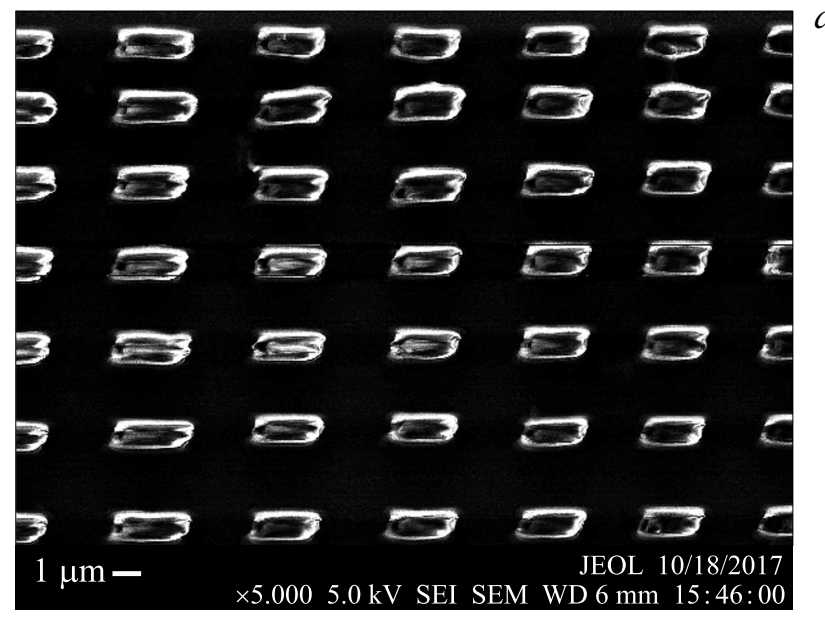

IIIIIIIIIIIIIIIIIIIIIIIIIIIIIIII ${ }^{b}$

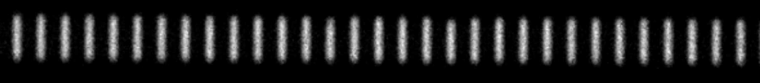
IIIIIIIIIIIIIIIIIIIIIIIIIIIIIIII 1IIIIIIIIIIIIIIIIIIIIIIIIIIIIIII IIIIIIIIIIIIIIIIIIIIIIIIIIIIIIII IIIIIIIIIIIIIIIIIIIIIIIIIIIIIIII|

Рис. 3. (а) Морфология полимерного шаблона для создания массива плазмонных наноантенн, измеренная с помощью сканирующего электронного микроскопа (SEM). Полимерный шаблон имеет вид массива параллелепипедов с длиной $2.3 \mu \mathrm{m}$, шириной $1 \mu \mathrm{m}$ и периодом по оси вдоль длинной стороны антенны $4 \mu \mathrm{m}$, а вдоль короткой $-3 \mu \mathrm{m}$. (b) Изображение в темнопольном оптическом микроскомассива плазмонных наноантенн. Плазмонные наноантенны являются золотыми параллелепипедами с длиной $2.3 \mu \mathrm{m}$, шириной $0.15 \mu \mathrm{m}$ и высотой $80 \mathrm{~nm}$. Период массива $4.3 \mu \mathrm{m}$ вдоль длинной стороны и $1.15 \mu \mathrm{m}$ вдоль короткой. 


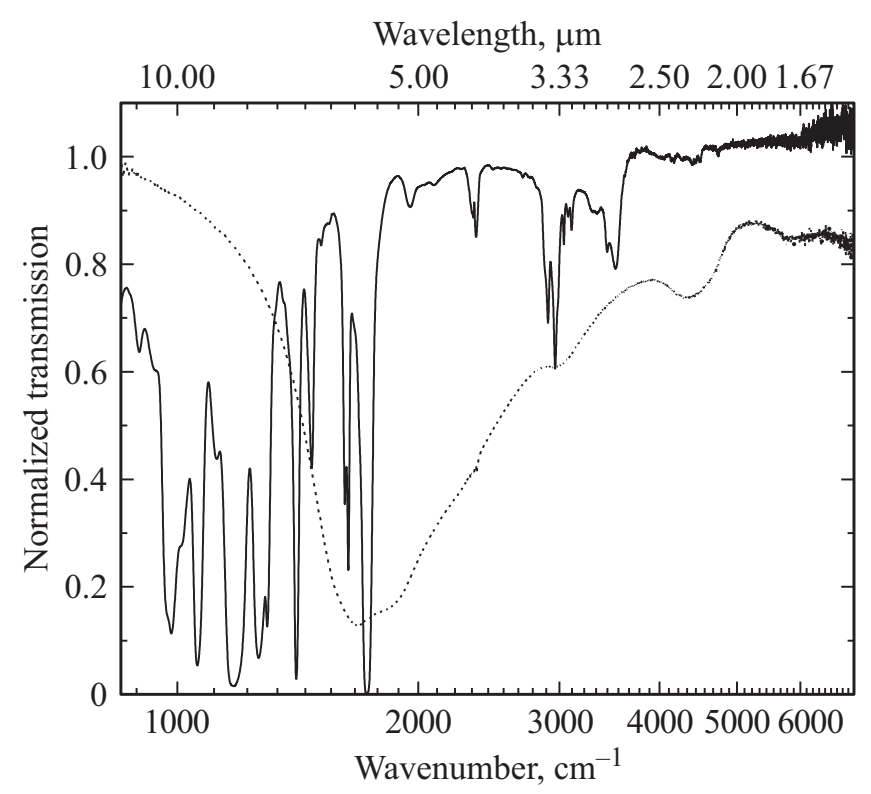

Рис. 4. Спектры пропускания олигомера РЕТTА и массива плазмонных наноантенн. Спектры измерены методами ИК фурье-спектроскопии. Сплошной линии соответствует спектр пропускания PETTA, пунктирной - массива плазмонных наноантенн с поляризацией излучения, направленной вдоль длинной стороны антенн.

спин-коатинга (spin-coating) с различными параметрами скорости и концентрации олигомера РЕТТА в растворе изопропилового спирта. После фотополимеризации олигомера толщина полученной полимерной пленки измерялась на атомно-силовом микроскопе Ntegra Spectra.

Спектры изготовленных плазмонных и полимерных структур регистрировались в режиме пропускания при помощи FTIR-спектрометра Bruker Vertex 80v с микроскопной приставкой Hyperion 2000 (диапазон частот $500-19000 \mathrm{~cm}^{-1}$, пространственное разрешение $\left.8 \mu \mathrm{m}\right)$ и ИК поляризаторами.

\section{Обсуждение}

Спектры пропускания олигомера РЕТTА и массива плазмонных наноантенн (рис. 4) измерены методом ИК фурье-спектроскопии. Минимумы в спектре пропускания олигомера PETTA соответствуют вращательноколебательным переходам в РЕТТА. Исследование относительной интенсивности пиков поглощения $\mathrm{C}=\mathrm{C}$-связей $\left(1618\right.$ и $\left.1635 \mathrm{~cm}^{-1}\right)$ и $\mathrm{C}=\mathrm{O}$-связей $\left(1721 \mathrm{~cm}^{-1}\right)$ в спектре поглощения РЕТТА позволяют сделать вывод о степени конверсии олигомера РЕТTА. Информация о степени конверсии важна при создании полимерных структур и шаблонов методами литографии.

Изменения относительных интенсивностей линий поглощения $\mathrm{C}=\mathrm{C}$-связей $\left(1618\right.$ и $\left.1635 \mathrm{~cm}^{-1}\right)$ и $\mathrm{C}=\mathrm{O}$-связей $\left(1721 \mathrm{~cm}^{-1}\right)$ в спектрах ИК поглощения РЕТТА при различных методах фотополимеризации не столь велики, что увеличивает погрешность при определении степени конверсии. Для улучшения чувствительности измерений предложено использовать эффект SEIRA.

Массив плазмонных наноантенн в форме параллелепипеда рассматривается как SEIRA-активная поверхность. Характерные размеры и период структуры были рассчитаны для возбуждения плазмонного резонанса вблизи $1700 \mathrm{~cm}^{-1}$. Данный массив возможно получить методами стереолитографии с последующей взрывной литографией. Пик возбуждения поверхностного плазмонного резонанса такого массива приходиться на $1700 \mathrm{~cm}^{-1}$ с поляризацией падающего излучения, направленного вдоль длинной стороны антенн. Ширина пика $1000 \mathrm{~cm}^{-1}$. При падении излучения с поляризацией, перпендикулярной длинной стороне антенн, возбуждения плазмонного резонанса не происходит.

\section{Заключения}

Экспериментально продемонстрированы возможности современной оптической литографии DLW, основанный на двухфотонной фемтосекундной лазерной полимеризации, и возможность улучшения пространственного разрешения с помощью STED-метода. Развита технология STED-DLW-стереолитографии. Данным методом изготовлены полимерные 2D- и 2.5D-структуры с латеральным размером менее $150 \mathrm{~nm}$. Полученные диэлектрические структуры являются шаблонами (при их дальнейшей металлизации) для изучения плазмонных эффектов (в частности, SEIRA).

Исследованы спектральные свойства массивов плазмонных золотых наноантенн, найдены длины волн основных дипольных резонансов (от 3 до $6 \mu \mathrm{m}$ ) и проведено сопоставление положения резонансов с их теоретическими значениями. Показаны возможности применения полученных плазмонных структур для исследования эффекта SEIRA в ИК диапазоне со STED-совместимым олигомером PETTA в качестве аналитического слоя.

Продемонстрирована возможность управления эффективностью возбуждения резонанса с помощью изменения направления линейной поляризации падающего ИК излучения. Несомненно, исследуемый эффект SEIRA найдет приложения в химии, биохимии и биофизике.

Авторы благодарны В.В. Сычеву, Д.В. Негрову (ЦКП МФТИ), Е.В. Кростылеву (ЦКП МФТИ) за ценные консультации и техническую помощь, а также Е.С. Жуковой за помощь в поляризационных измерениях. Технологическая часть работы выполнена при поддержке гранта РНФ № 15-19-00205, исследования спектральных свойств созданных структур - при поддержке гранта РНФ № 17-79-20418. Возможность проведения тонких оптических экспериментов дали гранты РФФИ № 18-32-01051 и 17-02-01408 и госзадание № $16.11575 .2018 / 10.11$. 


\section{Список литературы}

[1] Fleischmann M., Hendra P.J., McQuillan A.J. // Chem. Phys. Lett. 1974. V. 26. N 2. P. 163-166. doi 10.1016/00092614(74)85388-1

[2] Van Duyne R.P., Jeanmaire D.L. // J. Electroanalytical Chemestry. 1977. V. 84. N 1. P. 1-20. doi 10.1016/S00220728(77)80224-6

[3] Tsang J.C., Kirtley J.R., Bradley J.A. // Phys. Rev. Lett. 1979. V. 43. N 11. P. 772. doi 10.1103/PhysRevLett.43.772

[4] Zong C., Xu M., Xu L., Wei T., Xin Ma, Zheng X., Ren Hu, Bin Ren // Chem. Rev. 2018. V. 118. N 10. P. 4946-4980. doi 10.1021/acs.chemrev.7b00668

[5] Panneerselvam R., Liu G., Wang Y., Liu J., Ding S., Li J., Wu D., Tian Z. // Chem. Commun. 2018. V. 54. N 1. P. 10-25. doi 10.1039/C7CC05979E

[6] Hartstein A., Kirtley J.R., Tsang J.C. // Phys. Rev. Lett. 1980. V. 45. N 3. P. 201. doi 10.1103/PhysRevLett.45.201

[7] Neubrech F., Huck C., Weber K., Pucci A., Giessen H. // Chem. Rev. 2017. V. 117. N 7. P. 5110-5145. doi 10.1021/acs.chemrev.6b00743

[8] Altissimo M. // Biomicrofluidics. 2010. V. 4. N 2. P. 026503. doi 10.1063/1.3437589.

[9] Pao Y.H., Rentzepis P.M. // Appl. Phys. Lett. 1965. V. 6. N 5. P. 93-95. doi 10.1063/1.1754182

[10] Sun H.B., Kawata S. NMR. 3D Analysis. Photopolymerization. Advances in Polymer Science. Berlin, Heidelberg: Springer, 2004. V. 170. doi 10.1007/b94405

[11] Baldacchini T. Three-dimensional Microfabrication Using Two-photon Polymerization: Fundamentals, Technology and Applications. William Andrew, 2015.

[12] Hell S.W. // Angewandte Chemie International Edition. 2015. V. 54. N 28. P. 8054-8066. doi 10.1002/anie.201504181

[13] Wildanger D., Patton B.R., Schill H., Marseglia L., Hadden J.P., Knauer S., Schönle A., Rarity J.G., O'Brien J.L., Hell S.W., Smith J.M. // Advanced Materials. 2012. V. 24. N 44. P. OP309-OP313. doi 10.1002/adma.201203033

[14] Gansel J.K., Thiel M., Rill M.S., Decker M., Bade K., Saile V., von Freymann G., Linden S., Wegener M. // Science. 2009. V. 325. N 5947. P. 1513-1515. doi 10.1126/science.1177031

[15] Bagheri S., Weber K., Gissibl T., Weiss T., Neubrech F., Giessen H. // ACS Photonics. 2015. V. 2. N 6. P. 779-786. doi 10.1021/acsphotonics.5b00141

[16] Kaschke J., Wegener M. // Optics Letters. 2015. V. 40. N 17. P. 3986-3989. doi 10.1364/OL.40.003986

[17] Vitukhnovsky A.G., Chubich D.A., Eliseev S.P., Sychev V.V., Kolymagin D.A., Selyukov A.S. // J. Russian Laser Research. 2017. V. 38. N 4. P. 375-382. doi 10.1007/s10946-017-9656-2

[18] Kolymagin D.A., Zvagelsky R.D., Chubich D.A., Vitukhnovsky A.G. // KnE Energy \& Physics. 2018. V. 3. N 3. P. 14-22. doi 10.18502/ken.v3i3.2009 